\title{
INFODEMIC MONIKERS IN SOCIAL MEDIA DURING COVID-19 PANDEMIC
}

\author{
Jeevan Bhattal', Sharmistha Sharma2, Shashi Kandel3, Roshan Nepal ${ }^{4}$ \\ 1. ASEAN Institute for Health Development, Mahidol University, Thailand \\ 2. Think Aloud P Ltd, Kathmandu, Nepal \\ 3. Prince of Songkla University, Thailand \\ 4. The University of Adelaide, Australia
}

Correspondence: gwan.ph@gmail.com

\begin{abstract}
Social media is a common platform that enables its users to share opinions, personal experiences, perspectives with one another instantaneously, globally. It has played a paramount role during pandemics such as COVID-19 and unveiled itself as a crucial means to communicate between the sources and the individuals. However, it also has become a place to disseminate misinformation and fake news rapidly. Infodemic, a plethora of information, some authentic some not makes it even harder to general people to receive factual and trustworthy information when required, has grown to be a major risk to public health and social media is developing as a trendy platform for this infodemic. This commentary aims to explore how social media has affected the current situation. We also aim to share our insight to control this misinformation. This commentary contributes to evolving knowledge to counter fake news or health-related information shared over various social media platforms.
\end{abstract}

\section{KEYWORDS}

COVID-19, Pandemic, Infodemic, Social Media

\section{INTRODUCTION}

The Coronavirus disease (COVID-19) is an infectious disease transmitted primarily through respiratory droplets or contact with the contaminated surface, caused by newly discovered coronavirus. The number of confirmed cases infected with this novel disease is expanding rapidly. The pandemic has changed people's regular lifestyles. Developed countries have slowly started to move back to their new normal life with various public health measures whereas the underdeveloped and developing countries are still heavily relying on lockdown along with the various public health measures. [1]

Globally, the Internet is an increasingly valuable forum for acquiring the information on the novel coronavirus (COVID-19) pandemic. [2,3] Online and social data analysis approach is termed Infodemiology. [4] 'Infodemic monikers' are described as significantly inaccurate information that results in errors of perception, false news, racial episodes or any other types of misleading information circulated on the Internet. [5] Such monikers may have a major impact on dialog about public health and even relate to xenophobia. $[5,6]$

\section{SOCIAL MEDIA AND HEALTH INFODEMIC}

Unlike in past pandemics, the population of developing countries now has widespread access to the internet. At present, 4.5 billion people worldwide have internet access with $7.1 \%$ annual growth in the number of global internet users. Amid the pandemic and subsequent lockdown and with unemployment, people tend to spend more time on the internet and there has been a surge in social media usage such as YouTube, Facebook, Twitter, Instagram, TikTok, WhatsApp etc. In a recent study, people when asked from where they got the news/information, 36 percent 
named a news organization website or applications; 35 percent said social media; 20 percent recalled a search engine; 15 percent indicated a news organization email, text, or alert; 9 percent said it was another source, and 7 percent named a family member email or text. [7] The sources of the information are varied, not limited to popular search engines, and recognized health database resources, but also include social media. However, it may also contain fake news, fabricated stories, and disinformation, this can spread through social networks even faster than true information. For example, the antivaccine discourse on social media has been referred to as one of the major factors for parents' refusal to vaccinate their children [8]. Rumors spread through social media during the Ebola outbreak in West Africa were one of the forces that hindered disease control efforts. $[9,10]$

Social media has become an indispensable medium to communicate between the authorities and the people. In the middle of this pandemic, social media are deluged with COVID-19-related content. Hoax or fake news about COVID-19 relayed in social media is currently termed as health misinformation. World Health Organization (WHO) mentioned it is concentrating its effort to curtail the spread of the COVID-19 outbreak. But a global epidemic of misinformation-widening briskly via social media platforms and other outlets-introduces a major obstacle for public health. "We're not just fighting an epidemic; we're fighting an infodemic", said WHO Director-General Tedros Adhanom Ghebreyesus. [11, 12]

Recently WHO has cautioned people against infodemic i.e. a situation when 'misinformation' spreads rapidly thereby affecting thinking and leading to panic, stigma, along with irrational behavior. For instance, within a week after the identification of the first case of the novel coronavirus in December 2019 in China, conspiracy theories about the origin of the virus began to spread through every social media channel: that the virus was a leaked bio weapon of the Wuhan Institute of Virology, that the government of China planned the outbreak, that the locals' habit of eating bat soup was the cause of the outbreak. [13] This fake fabricated news contributed to heightened racism, xenophobic violence, and discrimination against Chinese people worldwide. With the current increase in the number of new cases, misinformation is making the work of health professionals more difficult. When the WHO declared apublic health emergency of international concern, the WHO also launched the WHO Information Network for Epidemics (EPI-
WIN). The goal of EPI-WIN is to share customized information with specific target groups, yet the WHO encountered a massive 'infodemic'.[14] In February 2020, at the Munich Security Conference, WHO Director-General Tedros Adhanom Ghebreyesus said: "Fake news spreads faster and more easily than this virus, and is just as dangerous" and urged prominent social media firms to act on false information and rumors about the pandemic. [14]

Additionally, it is difficult to identify which pages and media have credible health information. The most frequently accessed website is Google, and most Google search queries are all related to coronavirus, with keywords such as coronavirus tips, updates, symptoms, news, prevention. [15] A quarter of videos viewed on YouTube had various misleading information, yet most of these videos reached millions of viewers globally. [16] In Venezuela, people received false WhatsApp messages stating, "Stay home, the UN will bring you food". Immediately the UN released a press release denying the information. [17] In India, after the Tablighi Jamaat gathering, Muslims were blamed for spreading the virus, and eleven people were hospitalized after consuming a poisonous plant in Andhra's Chittoor district after watching a Tit-Tok video that claimed the plant to be a homemade remedy for COVID-19. [18] Moreover, some information about COVID-19 provided on the social media platform might be difficult to understand, while misleading claims from politicians, head of states, religious leaders and commentaries based on the assumptions but not scientifically proven facts and evidence may lead to disaster and a massive surge in the risk of COVID-19 in the population. [19]

\section{CONCLUSION}

It is crucial to address the issue of fake news without undermining the benefits of social media. Governments should promote news literacy and strong professional journalism in their societies. Social media platforms should invest in technologies that spot fake news and ban or delete false news immediately which has not been published or denied by an authentic source. The platforms should stop financial benefits for those who gain from disinformation. Awareness programs focused on myths about COVID-19 must take place regularly. People must be encouraged via various traditional media like radios, television, SMS alert to only trust information shared by their health ministry and other genuine platforms. Helpline numbers for people to clarify their doubts and concerns 
about COVID-19. Public figures need to think carefully before posting any content as people believe their instruction.

The infodemic is as real as Covid-19. Hence, an accurate information-seeking behavior during this pandemic is vital for health-related behavior change and may strengthen infection prevention and control. Individuals should follow a diversity of news sources and be skeptical of what they read and watch. Everyone should be critical while looking at social media, should report the false information to the platform, check the fact and verify the information before sharing, ask the person who shared the false news to remove it and finally make more noise than people who share false information.

\section{REFERENCES}

1. Sharma S, Bhatta J. Public health challenges during the COVID-19 outbreak in Nepal: a commentary. Journal of Health Research. 2020 Jun 5..

2. Bento Al, Nguyen T, Wing C, Lozano-Rojas F, Ahn Y-Y, Simon KJPotNAoS. Evidence from internet search data shows information-seeking responses to news of local COVID-19 cases. 2020;117(21):11220-2.

3. Rovetta A, Bhagavathula ASJm. Global Infodemiology of COVID-19: Focus on Google web searches and Instagram hashtags. 2020.

4. Eysenbach GJAjopm. Infodemiology and infoveillance: tracking online health information and cyberbehavior for public health. 2011;40(5):S154-S8.

5. Abd-Alrazaq A, Alhuwail D, Househ M, Hamdi M, Shah ZJJomlr. Top concerns of tweeters during the COVID19 pandemic: infoveillance study. 2020;22(4):e19016.

6. Rovetta A, Bhagavathula ASJJph, surveillance. Covid19-related web search behaviors and infodemic attitudes in italy: Infodemiological study. 2020;6(2):e19374.

7. Mitchell A, Gottfried J, Shearer E, LU K. How Americans encounter, recall, and act upon digital news. Pew Research Center. 2017.
8. Bode L, Vraga EKJHc. See something, say something: Correction of global health misinformation on social media. 2018;33(9):1131-40.

9. Broniatowski DA, Jamison AM, Qi S, AlKulaib L, Chen T, Benton A, et al. Weaponized health communication: Twitter bots and Russian trolls amplify the vaccine debate. 2018;108(10):1378-84.

10. Jones B, Elbagir NJCW. Are myths making the Ebola outbreak worse. 2014.

11. Zarocostas JJTL. How to fight an infodemic. 2020;395(10225):676.

12. COVID W. PHEIC Global research and innovation forum: towards a research roadmap. 2019.

13. Monitoring BJBT, Producer) Retrieved. China coronavirus: Misinformation spreads online about origin and scale. 2020;5(02):2020.

14. Adhanom-Ghebreyesus T, editor Munich security conference. Munich: World health organization https://www who int/dg/speeches/detail/munichsecurity-conference; 2020.

15. Morahan-Martin JMJC, Behavior. How internet users find, evaluate, and use online health information: a cross-cultural review. 2004;7(5):497-510.

16. Li HO-Y, Bailey A, Huynh D, Chan JJBGH. YouTube as a source of information on COVID-19: a pandemic of misinformation? 2020;5(5):e002604.

17. UN News. First Person: Countering COVID-19 misinformation in Venezuela. [Online].2020 [cited 2020 June 15]. Available at: https://news.un.org/en/story/2020/06/1065682

18. The News Minute. 11 in AP hospitalised after following TikTok poisonous 'remedy' for COVID-19. [Online].2020 [cited 2020 June 15]. Available at: https://www.thenewsminute.com/article/11-aphospitalised-after-following-tiktok-poisonous-remedycovid-19-122136

19. Tangcharoensathien $\mathrm{V}$, Calleja N, Nguyen T, Purnat T, D'Agostino M, Garcia-Saiso S, et al. Framework for managing the COVID-19 infodemic: methods and results of an online, crowdsourced WHO technical consultation. 2020;22(6):e19659. 